\title{
Dressed tunneling approximation for electronic transport through molecular transistors
}

\author{
R. Seoane Souto, ${ }^{*}$ A. Levy Yeyati, A. Martín-Rodero, and R. C. Monreal \\ Departamento de Física Teórica de la Materia Condensada, and Condensed Matter Physics Center (IFIMAC) and Instituto Nicolás Cabrera, \\ Universidad Autónoma de Madrid E-28049 Madrid, Spain
}

(Received 8 November 2013; revised manuscript received 24 January 2014; published 14 February 2014)

\begin{abstract}
A theoretical approach for the nonequilibrium transport properties of nanoscale systems coupled to metallic electrodes with strong electron-phonon interactions is presented. It consists of a resummation of the dominant Feynman diagrams from the perturbative expansion in the coupling to the leads. We show that this scheme eliminates the main pathologies found in previous simple analytical approaches for the polaronic regime. The results for the spectral and transport properties are compared with those from several other approaches for a wide range of parameters. The method can be formulated in a simple way to obtain the full counting statistics. Results for the shot and thermal noise are presented.
\end{abstract}

DOI: 10.1103/PhysRevB.89.085412

PACS number(s): 73.63.-b, 71.38.-k, 72.70.+m

\section{INTRODUCTION}

The effect of localized vibrations (phonons) in the electronic transport properties of nanoscale devices is attracting increasing attention (for a review see Ref. [1]). Such effects have been identified in different systems like atomic contacts and atomic chains [2], semiconducting quantum dots [3], carbon nanotubes [4], and other molecular junctions [5-7]. Other systems in which a strong electron-phonon coupling can lead to polaronic effects dominating the electronic transport are organic semiconductors [8,9].

In spite of this variety, from the theoretical point of view, all these situations can be qualitatively described by the rather simple Anderson-Holstein model. This model consists of a single resonant electronic level coupled to fermionic leads and to a localized phonon mode [10]. Even in the more simple spinless case, this model corresponds to a nontrivial strongly correlated system in an out-of-equilibrium situation. This model can be regarded as "paradigmatic" of an electronic system interacting with bosonic excitations. For instance, the same model was proposed by Langreth to describe the problem of photoemission through core-holes in metals [11].

This model has been extensively analyzed by different theoretical approaches [12-15] but there is still no exact solution available except for some limiting cases. Comparison with numerically exact methods like numerical renormalization group or quantum Monte Carlo is possible only for a certain range of parameters both for equilibrium [16-19] and more recently for nonequilibrium situations [20-23]. Results from functional renormalization group are also available [24].

Within this model one can distinguish between two different regimes depending on the strength of the electron-phonon coupling. For sufficiently weak coupling a lowest-order perturbation theory is applicable [25-27]. This situation is suitable to describe the case of atomic contacts and atomic chains [28,29]. As the electron-phonon coupling increases, higher-order diagrams, including vertex corrections, become of importance as discussed in Ref. [30]. In the opposite regime, the so-called polaronic regime, perturbation theory breaks down and other type of approaches are necessary [31-35].

*ruben.seoane@uam.es
The analysis of the transport properties of this model has been more recently extended to the case of noise and, more generally, to its full counting statistics (FCS). These studies have been mainly restricted to the perturbative regime [36-39]. Although there exist some studies of the noise properties in the polaronic regime [40,41] it is desirable to develop simple methods to analyze the crossover from the perturbative to the polaronic case.

Two simple approximations have been proposed to describe the polaronic regime: the so-called single-particle approximation (SPA) and the polaron tunneling approximation (PTA). Both approaches correspond to simple decoupling schemes which allow an analytical evaluation of the electronic Green functions. This simplicity has allowed, for instance, to extend PTA to analyze the transient behavior of this model yielding results in remarkably good agreement with numerically exact ones [42]. In spite of their several advantages both approximations exhibit some pathological features. This is particularly noticeable in their spectral properties at low frequencies (SPA) and high frequencies (PTA). Although there exist other methods to describe this polaronic regime based either on the equation of motion technique $[31,43]$ or other diagrammatic techniques [44-47], these methods require a more involved numerical evaluation. These methods are therefore not easy to extend to more complex situations like the calculation of the FCS or the analysis of the transient behavior in the nonstationary case.

The aim of the present work is to develop a simple method for describing the crossover region from the polaronic to the perturbative regime. Ideally this method should recover the good features of SPA and PTA commented above while eliminating their pathologies. By analyzing the exact perturbation series with respect to the tunneling to the leads we identify a family of diagrams which gives the dominant contribution in the polaronic regime and which can be summed up exactly. We will denote this approach the dressed tunneling approximation (DTA) [48] as it corresponds to dressing the leads self-energy with the polaronic cloud. In spite of being derived for describing the polaronic regime we show that this approximation gives a reasonable description of the crossover region while exhibiting an increasing deviation from the perturbative results in the corresponding limit. 
The article is organized as follows: in Sec. II we introduce the model Hamiltonian and the basic Green functions formalism which allows us to calculate the different electronic and transport properties. In Sec. III we analyze the diagrammatic expansion of the relevant Green functions in the polaronic limit and briefly introduce the known simple approximations like PTA and SPA. Section IV is devoted to introduce the DTA discussing the arguments for its derivation and giving the main expressions for the system Green functions. The corresponding results are described in Sec. V where the spectral densities are compared with other approaches. In this section we also analyze the DTA results for the transport properties like the current, the differential conductance, and the noise. Finally, we summarize the main results of this work in Sec. VI.

\section{MODEL AND BASIC THEORETICAL FORMULATION}

We consider the simplest spinless Anderson-Holstein model in which a single electronic level is coupled to a localized vibrational mode. Electrons can tunnel from this resonant level into a left $(\mathrm{L})$ and a right electrode $(\mathrm{R})$. We shall generically refer to this central region, which can represent a molecule, an atomic chain or a quantum dot, as the "dot" region. The corresponding Hamiltonian is given by $H=H_{\text {leads }}+H_{\text {dot }}+H_{T}$, with (in natural units, $\hbar=k_{B}=$ $\left.e=m_{e}=1\right)$

$$
H_{\mathrm{dot}}=\left[\epsilon_{0}+\lambda\left(a^{\dagger}+a\right)\right] d^{\dagger} d+\omega_{0} a^{\dagger} a,
$$

where $\epsilon_{0}$ is the bare electronic level, $\lambda$ is the electron-phonon coupling constant and $\omega_{0}$ is the frequency of the localized vibration. The electron (phonon) creation operator in the dot is denoted by $d^{\dagger}\left(a^{\dagger}\right)$. On the other hand, $H_{\text {leads }}=\sum_{j k} \epsilon_{j k} c_{j k}^{\dagger} c_{j k}$ corresponds to the noninteracting leads Hamiltonian $(j \equiv$ $L, R)$, where $\epsilon_{j k}$ are the leads electron energies and $c_{j k}^{\dagger}$ are the corresponding creation operators. The bias voltage applied to the junction is imposed by shifting the chemical potential of the electrodes $V=\mu_{L}-\mu_{R}$. The tunneling processes are described by

$$
H_{T}=\sum_{k}\left(t_{L k} c_{L k}^{\dagger} d+t_{R k} c_{R k}^{\dagger} d+\text { H.c. }\right),
$$

where $t_{j k}$ are the tunneling amplitudes.

To address the polaronic regime it is convenient to perform the so-called Lang-Firsov unitary transformation [49], which allows us to eliminate the linear term in the electron-phonon coupling [50],

$$
\tilde{H}=S H S^{\dagger}, \quad S=e^{g d^{\dagger} d\left(a^{\dagger}-a\right)}, \quad g=\frac{\lambda}{\omega_{0}} .
$$

Using this transformation

$$
\tilde{H}_{\mathrm{dot}}=\tilde{\epsilon} d^{\dagger} d+\omega_{0} a^{\dagger} a,
$$

where $\tilde{\epsilon}=\epsilon_{0}-\lambda^{2} / \omega_{0}$. The tunneling Hamiltonian is transformed as

$$
\tilde{H}_{T}=\sum_{k}\left(t_{L k} c_{L k}^{\dagger} X d+t_{R k} c_{R k}^{\dagger} X d+\text { H.c. }\right) .
$$

where $X=\exp \left[g\left(a-a^{\dagger}\right)\right]$ is the phonon cloud operator. On the other hand, the free leads Hamiltonian remains invariant. For later use it is useful to introduce the tunneling rates
$\Gamma_{j}=\operatorname{Im} \sum_{k}\left|t_{j k}\right|^{2} /\left(\omega-i 0^{+}-\epsilon_{j k}\right)$, which are approximated by constants in the so-called wideband approximation.

To deal with the transport properties of this model it is convenient to use the Keldysh nonequilibrium formalism [51]. The basic quantity required to calculate the electronic and transport properties are the dot Green functions

$$
G^{\alpha \beta}\left(t, t^{\prime}\right)=-i\left\langle T_{\mathcal{C}}\left\{X(t) d(t) X^{\dagger}\left(t^{\prime}\right) d^{\dagger}\left(t^{\prime}\right)\right\}\right\rangle,
$$

where $T_{\mathcal{C}}$ is the time ordering operator in the Keldsyh contour and $\alpha, \beta \equiv+,-$ denote the different branches of the contour.

A slight modification in the Keldysh formulation allows us to address directly the noise properties of the system and, more generally, its FCS [52,53]. This is achieved by introducing a "counting-field" $v$, which changes sign on the two branches of the contour and which enters as a phase factor modulating the tunnel Hamiltonian. As the current is conserved in our two terminal device one can choose to introduce the counting field in either the left or the right tunneling term. For definiteness we choose to include it in the left and accordingly we define

$$
\tilde{H}_{T}^{v}=\sum_{k}\left(e^{i v / 2} t_{L k} c_{L k}^{\dagger} X d+t_{R k} c_{R k}^{\dagger} X d+\text { H.c. }\right),
$$

and the Keldysh Green functions (GFs) in the presence of the counting field are

$$
G^{\alpha \beta(\nu)}\left(t, t^{\prime}\right)=-i\left\langle T_{\mathcal{C}}\left\{X(t) d(t) X^{\dagger}\left(t^{\prime}\right) d^{\dagger}\left(t^{\prime}\right) e^{-i \int_{\mathcal{C}} \tilde{H}_{T}^{v}(\tau) d \tau}\right\}\right\rangle_{0},
$$

where the subscript 0 indicates averaging over the states of the uncoupled Hamiltonians $\tilde{H}_{\text {dot }}$ and $H_{\text {leads }}$. More generally, the FCS can be obtained from a cumulant generating function (CGF),

$$
\chi(v)=\left\langle T_{\mathcal{C}} e^{-i \int_{c} d t \tilde{H}_{T}^{v}(t)}\right\rangle_{0} .
$$

Formally $\chi(\nu)$ can be expanded as $\chi(\nu)=\sum_{q} e^{i q v} P_{q}$, where $P_{q}$ is the probability of a charge $q$ being transferred through the system. So the cumulants can be computed using

$$
\left\langle\delta^{n} q\right\rangle=\left.(-i)^{n} \frac{\partial^{n}}{\partial \nu^{n}} \ln \chi(\nu)\right|_{\nu=0} .
$$

In a stationary situation the first cumulant $(n=1)$ in (10) corresponds to the mean current

$$
I_{L}=\int \frac{d \omega}{2 \pi} \sum_{k}\left[t_{L k} g_{L k}^{+-}(\omega) G^{-+}(\omega)-t_{L k}^{*} g_{L k}^{-+}(\omega) G^{+-}(\omega)\right],
$$

where $g_{j}^{\alpha \beta}$ are the isolated leads GFs. A symmetrized expression of the current involving only the dot spectral density can be deduced using current conservation, leading to [54]

$$
I=\frac{8 \Gamma_{L} \Gamma_{R}}{\Gamma} \int d \omega\left[f_{L}(\omega)-f_{R}(\omega)\right] A(\omega),
$$

where $\Gamma=\Gamma_{L}+\Gamma_{R}$ and the spectral function $A(\omega)=$ $-\operatorname{Im}\left(G^{R}(\omega)\right) / \pi, G^{R}$ being the retarded dot Green function.

On the other hand, the second cumulant corresponds to the current noise and can be written as

$$
\begin{aligned}
S_{L}= & i \int \frac{d \omega}{2 \pi} \sum_{k} \frac{\partial}{\partial \nu}\left[t_{L k} G^{-+(\nu)}(\omega) g_{L k}^{+-}(\omega) e^{i \nu}\right. \\
& \left.-t_{L k}^{*} G^{+-(\nu)}(\omega) g_{L k}^{-+}(\omega) e^{-i \nu}\right]\left.\right|_{\nu=0} .
\end{aligned}
$$




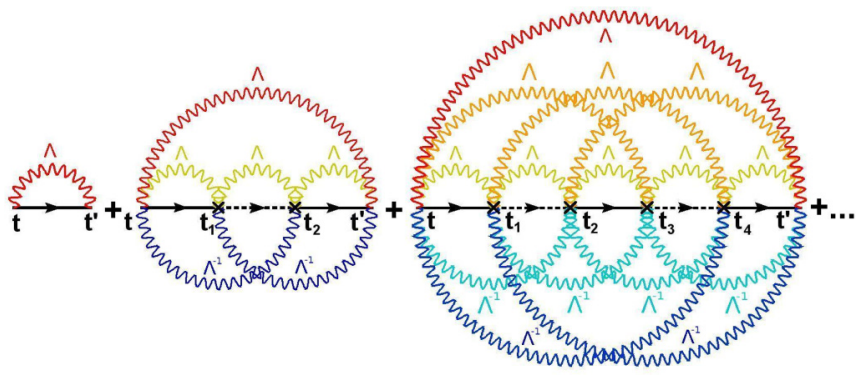

FIG. 1. (Color online) Lowest-order Feynman diagrams of the perturbative expansion in the tunneling Hamiltonian. The solid lines represents the free dot GF $\left(G_{0}\right)$, dashed lines represent the free lead GF $\left(g_{k}, k=L, R\right)$, the wavy lines denote the polaron correlator $(\Lambda)$ and the crosses the hopping events.

Symmetrized expressions for the noise in different approximations are given in Appendix A.

It is also convenient to define the unperturbed polaron correlator in Keldysh space,

$$
\Lambda^{\alpha \beta}\left(t, t^{\prime}\right)=\left\langle T_{C} e^{g\left(a(t)-a^{\dagger}(t)\right)} e^{-g\left(a\left(t^{\prime}\right)-a^{\dagger}\left(t^{\prime}\right)\right)}\right\rangle_{0} .
$$

\section{DIAGRAMMATIC EXPANSIONS IN THE POLARONIC REGIME}

An exact solution to the problem of determining the GFs entering in the calculations of the various transport properties is still unknown. In the polaronic regime a perturbative expansion in the hopping to the leads would be appropriate. The lowestorder diagrams in this expansion are shown in Fig. 1.

Therefore, the natural starting point for studying the system in this regime is provided by the so-called atomic limit [55]. This is defined as the limit when the tunneling rates between the dot and the leads tend to zero. The Green functions in this limit can be calculated exactly and correspond to the zero-order term in the expansion depicted in Fig. 1, and thus its Keldysh components can be computed as $G^{(0) \alpha \beta}(\omega)=G_{0}^{\alpha \beta}(\omega) \otimes \Lambda^{\alpha \beta}(\omega)$, where $G_{0}^{\alpha \beta}$ are the bare dot GFs and $\otimes$ represents the convolution product. In frequency domain its retarded component has the form

$$
G^{(0) R}(\omega)=\sum_{k=-\infty}^{\infty} \frac{\alpha_{k} n_{0}+\alpha_{-k}\left(1-n_{0}\right)}{\omega-\tilde{\epsilon}+k \omega_{0}+i \eta},
$$

where $n_{0}$ represents the average occupation number of the level of the dot, $\eta$ is an infinitesimal, and $\alpha_{k}$ is a coefficient that, at finite temperature, can be written as

$$
\alpha_{k}=e^{-g^{2}\left(2 n_{p}+1\right)} I_{k}\left(2 g^{2} \sqrt{n_{p}\left(1+n_{p}\right)}\right) e^{k \beta \omega_{0} / 2},
$$

$I_{k}$ being the modified Bessel function of the first kind, which is symmetric in the $k$ argument $\left(I_{k}=I_{-k}\right)$, and $n_{p}$ is the Bose factor $1 /\left(e^{\beta \omega_{0}}-1\right)$ with $\beta=1 / T$. At zero temperature this coefficient can be simplified as

$$
\alpha_{k}=\left\{\begin{array}{ccc}
e^{-g^{2} \frac{g^{2 k}}{k !}} & \text { if } \quad k \geqslant 0 \\
0 & \text { if } \quad k<0
\end{array}\right.
$$

There exist in the literature two simple ways to include the effects of finite tunneling to the leads starting from the atomic

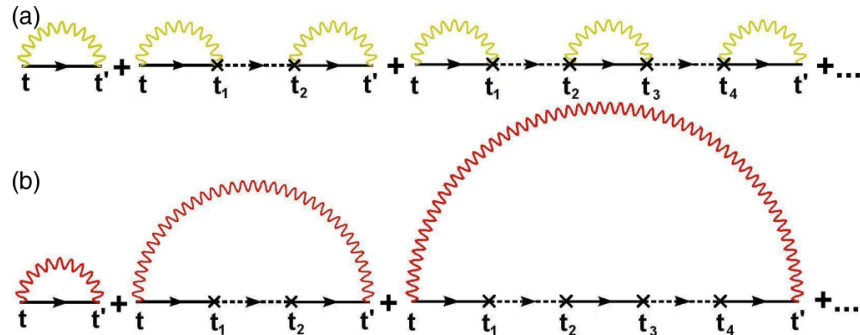

FIG. 2. (Color online) Feynman diagrams for (a) PTA and (b) DSPA. In both cases, the zero order corresponds to the atomic limit GF (15).

limit. These are the so-called polaron tunneling approximation (PTA) and the single-particle approximation (SPA), which are briefly described below.

Within PTA the phonons are assumed to be excited and deexcited instantaneously when the electrons tunnel from the leads to the dot [41]. Diagrammatically, this approximation corresponds to summing up the series depicted in Fig. 2(a), i.e., can be expressed in a Dyson-like equation in Keldysh space (for simplicity we concentrate in the $v=0$ case for the discussion within this and the next section)

$$
\mathbf{G}_{\mathrm{PTA}}=\mathbf{G}^{(0)}+\mathbf{G}^{(0)} \Sigma_{0} \mathbf{G}_{\mathrm{PTA}},
$$

where the self-energy $\boldsymbol{\Sigma}_{0}=\Sigma_{0 L}+\Sigma_{0 R}$, with

$$
\boldsymbol{\Sigma}_{0 j}(\omega)=i \Gamma_{j}\left[\begin{array}{cc}
2 f_{j}(\omega)-1 & -2 f_{j}(\omega) \\
-2\left(f_{j}(\omega)-1\right) & 2 f_{j}(\omega)-1
\end{array}\right] .
$$

This approximation provides a good description of the spectral properties at low energies and for situations close to half-filling [42]. In particular, it satisfies the Friedel sum rule (FSR) connecting the spectral density at zero energy with the dot charge, which implies that for the symmetric case $(\tilde{\epsilon}=0) A_{\mathrm{PTA}}(0)=1 / \pi \Gamma$ [34]. However, the spectral density at higher energies is somewhat pathological as it exhibits phonon sideband peaks of vanishing width but with a constant height. On the other hand, as has been shown in Ref. [42], this approximation provides a good description of the nonstationary evolution of the model at short time scales.

The SPA provides another simple picture of the polaronic regime. The general idea of this approximation is to directly decouple the electronic and the polaron degrees of freedom in the dot GFs. In the simplest form of this approximation [14] the retarded GF is given by

$$
G_{\mathrm{SPA}}^{R}(\omega)=\sum_{k=-\infty}^{\infty} \frac{\alpha_{k} n_{0}+\alpha_{-k}\left(1-n_{0}\right)}{\omega-\tilde{\epsilon}+k \omega_{0}+i \Gamma} .
$$

This expression is formally equivalent to broaden the poles of the atomic GF of Eq. (15) with the bare (frequency independent) tunneling rates to the leads. From this expression it is clear that this approximation does not satisfy the expected behavior at low frequencies as it does not fulfill the FSR nor reproduce the polaronic narrowing of the resonances close to the Fermi level. However, it does not exhibit the pathological behavior of the sideband peaks at higher energies characteristic 
of PTA and recovers the exact results in the limit of a fully occupied or fully empty dot $[12,56]$.

In a more transparent diagrammatic way the SPA decoupling scheme corresponds to the Feynman diagrams showed in Fig. 2(b) in which the bare dot Green function is dressed with the tunneling self-energy up to infinite order and then convoluted with the polaron correlator $\Lambda$ [45]. The resulting GFs are, however, not completely equivalent to the ansatz of Eq. (19). The Keldysh components within this diagrammatic SPA (DSPA) are given by

$$
\begin{aligned}
& G_{\mathrm{DSPA}}^{+-}(\omega)=-\sum_{k=-\infty}^{\infty} \alpha_{k} \frac{\Sigma_{0}^{+-}\left(\omega+k \omega_{0}\right)}{\mathfrak{D}\left(\omega+k \omega_{0}\right)} \\
& G_{\mathrm{DSPA}}^{-+}(\omega)=-\sum_{k=-\infty}^{\infty} \alpha_{-k} \frac{\Sigma_{0}^{-+}\left(\omega+k \omega_{0}\right)}{\mathfrak{D}\left(\omega+k \omega_{0}\right)},
\end{aligned}
$$

where $\mathfrak{D}(\omega)=(\omega-\tilde{\epsilon})^{2}+\Gamma^{2}$.

\section{DRESSED TUNNELING APPROXIMATION}

It is thus desirable to develop a simple approximation that would exhibit the properties of the PTA at low energies and of SPA at high energies. For this purpose let us analyze the full diagrammatic expansion illustrated in Fig. 1, taking as an example the second-order diagram represented again in Fig. 3(a). In the evaluation of this diagram there appear products of polaron correlators of the type $\Lambda\left(t, t_{1}\right) \Lambda^{-1}\left(t, t_{2}\right)$, where the time arguments $t_{1}$ and $t_{2}$ correspond to the exit and entrance of the electrons from the dot to the leads. As in the limit of strong electron-phonon coupling the lifetime of the electronic states in the dot is much larger than the one in the electrodes, it is then reasonable to make the approximation $\Lambda\left(t, t_{1}\right) \Lambda^{-1}\left(t, t_{2}\right) \sim 1$ [see Fig. 3(a)]. This can be more rigorously justified from the fact that in the wideband approximation the retarded leads self-energies are localized in time representation, i.e., $\Sigma_{0 j}^{R}\left(t, t^{\prime}\right) \propto \theta\left(t-t^{\prime}\right) \delta\left(t-t^{\prime}\right)$. With this prescription the diagrammatic expansion reduces to the one illustrated in Fig. 3(b), which can be evaluated exactly. One should notice that the cancellation of the "crossing" polaron lines in the diagrammatic expansion implies that vertex corrections can be neglected in this limit. An approximate

(a)

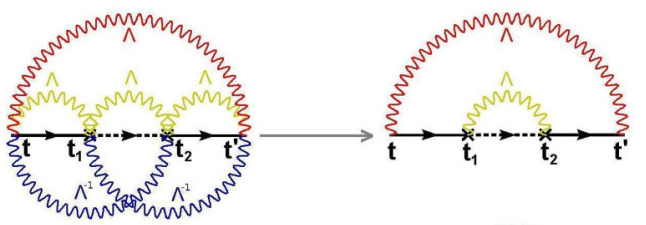

(b)

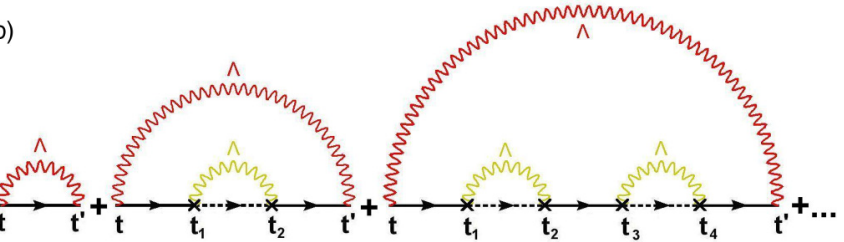

FIG. 3. (Color online) Feynman diagrams for the DTA approximation. Panel (a) indicates the simplifying approximation on the second-order diagram and panel (b) represents the diagrammatic series which is included within DTA. evaluation of these corrections in the $\lambda / \omega_{0}<1$ regime was undertaken in Ref. [45].

As can be observed, the resulting approximation is equivalent to dressing the leads self-energies within DSPA with the polaron correlators, i.e., $\tilde{\Sigma}^{\alpha \beta}(\omega)=\Sigma_{0}^{\alpha \beta}(\omega) \otimes \Lambda^{\alpha \beta}(\omega)$. In this way, the self-energy components can be written as

$$
\begin{aligned}
& \tilde{\Sigma}_{L, R}^{+-}(\omega)=\sum_{k=-\infty}^{\infty} \alpha_{k} \Sigma_{0 L, R}^{+-}\left(\omega+k \omega_{0}\right) \\
& \tilde{\Sigma}_{L, R}^{-+}(\omega)=\sum_{k=-\infty}^{\infty} \alpha_{-k} \Sigma_{0 L, R}^{-+}\left(\omega+k \omega_{0}\right) .
\end{aligned}
$$

Within the wideband approximation these self-energy components are purely imaginary quantities. The resulting GFs can then be straightforwardly evaluated as

$$
\begin{aligned}
& G_{\mathrm{DTA}}^{+-}(\omega)=-\sum_{k=-\infty}^{\infty} \alpha_{k} \frac{\tilde{\Sigma}^{+-}\left(\omega+k \omega_{0}\right)}{\tilde{\mathfrak{D}}\left(\omega+k \omega_{0}\right)} \\
& G_{\mathrm{DTA}}^{-+}(\omega)=-\sum_{k=-\infty}^{\infty} \alpha_{-k} \frac{\tilde{\Sigma}^{-+}\left(\omega+k \omega_{0}\right)}{\tilde{\mathfrak{D}}\left(\omega+k \omega_{0}\right)},
\end{aligned}
$$

where $\tilde{\Sigma}^{\alpha \beta}=\tilde{\Sigma}_{L}^{\alpha \beta}+\tilde{\Sigma}_{R}^{\alpha \beta}$ and

$$
\tilde{\mathfrak{D}}(\omega)=\left|\omega-\tilde{\epsilon}-\tilde{\Sigma}^{R}(\omega)\right|^{2},
$$

where

$$
\begin{aligned}
\tilde{\Sigma}^{R}(\omega)= & \sum_{\substack{k=-\infty \\
j=L, R}}^{\infty} \frac{i \alpha_{k}}{2 \pi} \int d \omega^{\prime}\left[\frac{\Sigma_{0 j}^{+-}\left(\omega^{\prime}\right)}{\omega+k \omega_{0}-\omega^{\prime}+i \eta}\right. \\
& \left.+\frac{\Sigma_{0 j}^{-+}\left(\omega^{\prime}\right)}{\omega-k \omega_{0}-\omega^{\prime}+i \eta}\right] .
\end{aligned}
$$

With these components, the spectral function can be determined as

$$
A_{\mathrm{DTA}}(\omega)=\frac{1}{2 \pi i}\left[G_{\mathrm{DTA}}^{+-}(\omega)-G_{\mathrm{DTA}}^{-+}(\omega)\right] .
$$

It should be noticed that a similar approach, but derived from a decoupling procedure within the equation of motion of the system GFs, was presented recently in Ref. [35]. We also point out that in all the preceding approximations the basic assumption of having a equilibrium phonon distribution was made for the evaluation of the polaron correlators.

A simpler version of this approximation can be obtained within the same spirit as in the SPA discussed in the previous section. Within this approximation [that we call approximated DTA (ADTA)], $G^{R}$ can be written as

$$
G_{\mathrm{ADTA}}^{R}(\omega)=\sum_{k=-\infty}^{\infty} \frac{\alpha_{k} n_{0}+\alpha_{-k}\left(1-n_{0}\right)}{\omega+k \omega_{0}-\tilde{\epsilon}-\tilde{\Sigma}^{R}\left(\omega+k \omega_{0}\right)} .
$$

From this expression it is clear that within this approximation the pole structure of the atomic limit is preserved but with a broadening determined by $\operatorname{Im} \tilde{\Sigma}^{R}$. As for large frequencies this effective broadening tends to $\Gamma$ one recovers the SPA result in this limit. However, this effective broadening is strongly reduced with respect to $\Gamma$ for low energies. In fact, for $\omega \rightarrow 0$ 
and for the symmetric case $\left|\operatorname{Im} \tilde{\Sigma}^{R}\right| \rightarrow e^{-g^{2}} \Gamma$, thus yielding the correct polaronic reduction in the resonance width at the Fermi level [43].

\section{RESULTS}

This section contains the predictions of the DTA for different physical quantities compared with other approaches.

\section{A. Spectral density}

We first analyze the results for the dot spectral density $A(\omega)$. Figure 4 shows the comparison of the DTA results for $A(\omega)$ with those from PTA and SPA for an electron-hole and left-right symmetric case in the polaronic regime. As can be observed, while the DTA and the PTA results tend to coincide at low energies (central resonance), they increasingly deviate at higher-order resonances. In contrast, for these higher-order resonances the DTA spectral density gradually converges to the SPA one. Therefore, as commented above, DTA contains the good features of both approximations but without their pathologies. The same conclusion is valid for the simpler ADTA method, as can be seen in Fig. 4.

In Fig. 5 we analyze the evolution of the spectral density with the applied bias voltage. As can be observed, the main effect of the applied bias is to gradually reduce the height of the phonon peaks. Remarkably, the DTA spectral density appears to evolve towards the SPA one, which is voltage independent (indicated by the dotted curve in Fig. 5). As large voltages correspond to high energies, one would expect that SPA should become exact in the limit $V \rightarrow \infty$. A similar convergence to the SPA is obtained in the limit $|\tilde{\epsilon}| \rightarrow \infty$, corresponding to the exact result for a fully empty or a fully dot case [56].

Another interesting property of DTA is that it reasonably describes the transition from the polaronic to the weak electron-phonon coupling regimes. This is illustrated in Fig. 6 in which the DTA spectral density is shown for two values of the parameter $g=1$ (lower panel) and $g=0.5$ (upper panel). For comparison we also show in these plots the corresponding results obtained by the interpolative self-energy approach (ISA) of Ref. [57] and extended to the nonequilibrium Holstein model in Ref. [44], which is constructed in order to interpolate

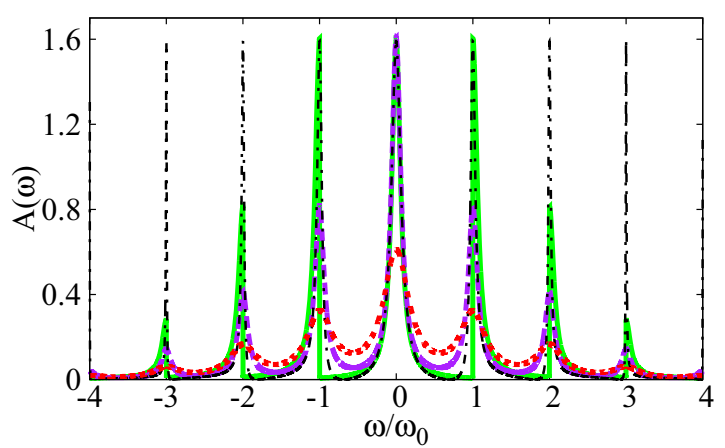

FIG. 4. (Color online) Spectral density for the symmetric case, zero bias voltage, and zero temperature with $\Gamma=0.2 \omega_{0}$ and $g=1.0$. The results correspond to the different approximations: DTA (full line, green or light gray), ADTA (full line, violet or dark gray), SPA (dotted line), and PTA (dashed line).

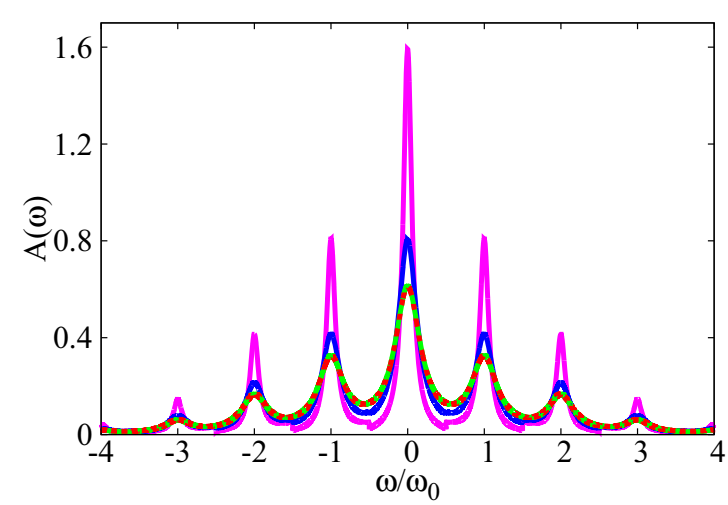

FIG. 5. (Color online) Evolution of the spectral density at zero temperature with increasing voltage within DTA. From top to bottom $V=1,3,9 \omega_{0}$. The other parameters as in lower panel of Fig. 6 . The dotted line corresponds to the SPA result, which is voltage independent.

between the second-order perturbation theory and the atomic limit. It is remarkable that the two approximations which are derived following such different criteria would so closely coincide in both regimes.

\section{B. Current and noise}

We analyze in this subsection the results from the DTA for several transport properties. As shown in the inset of Fig. 7 for moderate values of $g(g \sim 0.8)$ the currentvoltage characteristic starts to exhibit a steplike behavior.

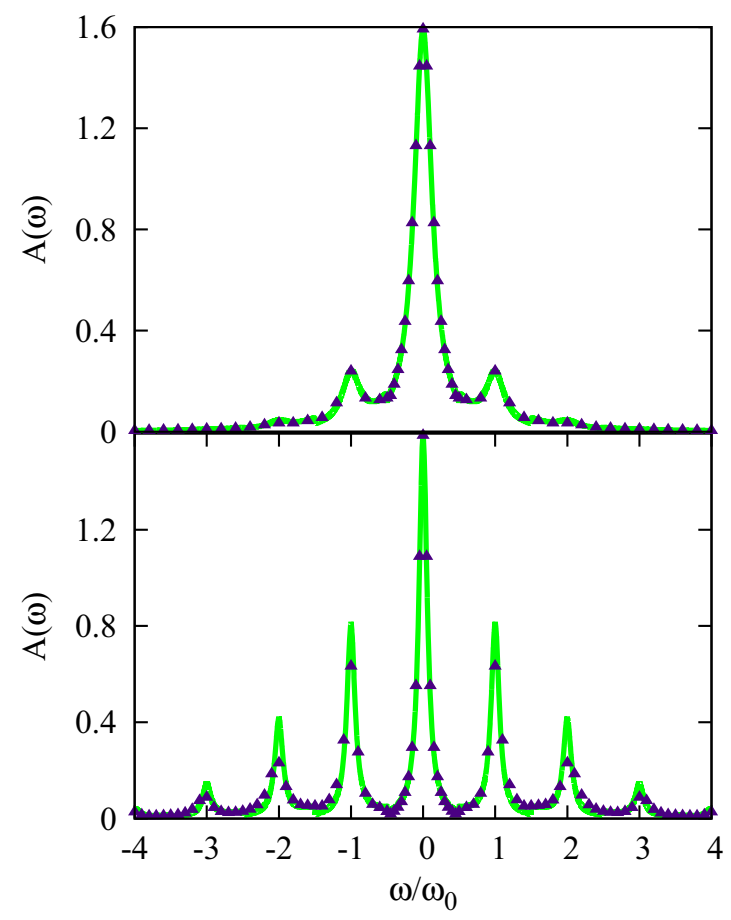

FIG. 6. (Color online) Spectral density at zero temperature in the DTA (full line) and the ISA (triangles) from Ref. [44] for the symmetric case with $\Gamma=0.2 \omega_{0}, V=\omega_{0}$ and two values of the coupling constant, $g=0.5$ (upper panel) and $g=1$ (lower panel). 


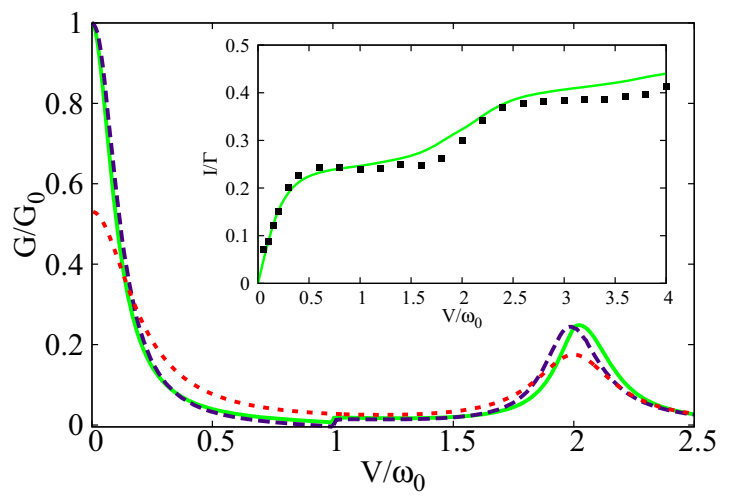

FIG. 7. (Color online) Zero-temperature differential conductance within DTA (full line, green or light gray), ISA (dotted line), and SPA (dots) for $\tilde{\epsilon}=0, \Gamma=0.1 \omega_{0}$, and $g=0.8$. The inset shows the current for the same parameters but with a finite temperature $T=0.04 \omega_{0}$ for comparison with diagrammatic Monte Carlo data (full squares) from Ref. [20].

For the electron-hole and left-right symmetric case shown in Fig. 7 the most pronounced features appear at $V \sim 2 n \omega_{0}$ $[22,41,44]$. It is interesting to note that the DTA results for the current quantitatively agree in this range of parameters with numerically exact results from diagrammatic MC calculations from Ref. [20], indicated by the symbols in the inset of Fig. 7. It should be also mentioned that, as shown in Appendix A, DTA fulfills the current conservation condition. This condition is trivially fulfilled by PTA where no inelastic processes are included but not, for instance, by DSPA. In the case of DSPA the violation of current conservation can be demonstrated explicitly (see Appendix A). However, as SPA and ADTA consist in an ansatz for the retarded GFs, the left and right currents cannot be calculated separately but just using the symmetrized expression of Eq. (12). Their nonconserving character can be inferred nevertheless from the violation of the FSR.

In order to have a more detailed analysis of the features in the IV characteristics it is convenient to calculate the differential conductance. This quantity is represented in the main panel of Fig. 7 for the same parameters as for the inset. We also show for comparison the corresponding results for the SPA and the ISA. Several features are worth noticing: (1) the zero bias conductance within DTA reaches the unitary limit as it corresponds to an electron-hole symmetric case. This condition, which is directly related to the FSR, is also fulfilled by ISA but not by SPA, which yields a smaller conductance value. (2) There appears a conductance step at $V \sim \omega_{0}$. This step corresponds to the onset of inelastic processes due to phonon emission, which is absent within SPA (neither PTA, not shown in Fig. 7, exhibits this feature), and (3) there appears a more pronounced feature at $V \sim 2 \omega_{0}$ corresponding to the sideband peaks in the spectral density. It should be noticed that the precise shape of this feature is extremely sensitive to the presence of a finite broadening of the logarithmic singularities in the real part of the electron self-energies. In fact, a finite broadening leads to a dip in the differential conductance at $V=2 \omega_{0}$ [35], which, however, tends to disappear as the broadening is reduced to zero.

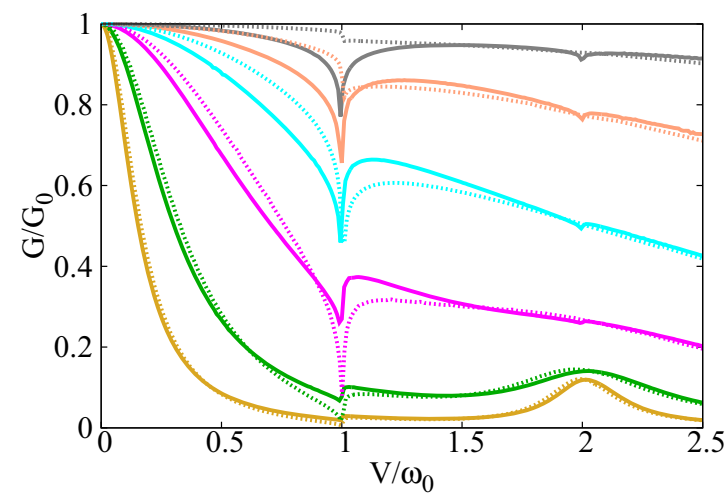

FIG. 8. (Color online) Zero-temperature conductance within DTA for $\tilde{\epsilon}=0, g=0.5$, and (from bottom to top) $\Gamma=0.1,0.2$, $0.5,1.0,2.0$, and $4.0 \omega_{0}$. The dashed lines show the corresponding results for ISA.

The inelastic features at $V \sim \omega_{0}$ become more pronounced as $\Gamma$ is increased. This is illustrated in Fig. 8, where the conductance is shown for fixed $g$ and increasing values of $\Gamma$. An interesting issue, which has been addressed repeatedly in the literature, is the transition from a step up to a step down in the conductance at the inelastic threshold. For instance, this transition was analyzed in the perturbative regime for the electron-phonon coupling in Refs. [28,29]. As shown in Fig. 8 the DTA fairly reproduces the step-up feature at small values of $\Gamma$ but the transition to the step-down behavior at large $\Gamma$ is somewhat masked by the presence of the logarithmic singularity in the real part of the electron self-energy. For comparison we show in Fig. 8 the corresponding results obtained with ISA, which by construction reproduces the expected step-down feature in the large $\Gamma$, in agreement with perturbation theory in $g$.

The behavior of the differential conductance as $\tilde{\epsilon}$ is varied, as shown in Fig. 9. It is interesting to analyze the evolution of the features at the inelastic threshold $V=\omega_{0}$. As can be

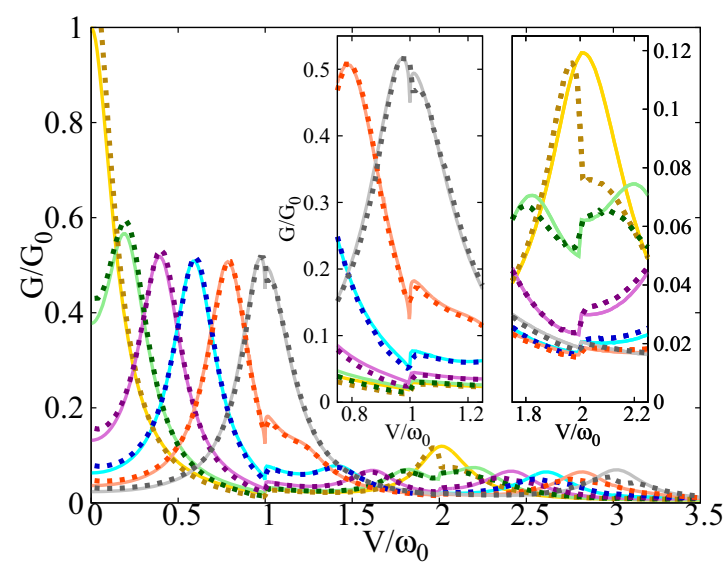

FIG. 9. (Color online) Zero-temperature conductance within DTA for $g=1, \Gamma=0.1 \omega_{0}$, and (from left to right) increasing values of $\tilde{\epsilon}=0.0,0.1,0.2,0.3,0.4$, and $0.5 \omega_{0}$. The dashed lines show the corresponding results for the EOM method of Ref. [43]. The insets correspond to a blow up of the first and second phonon resonances. 


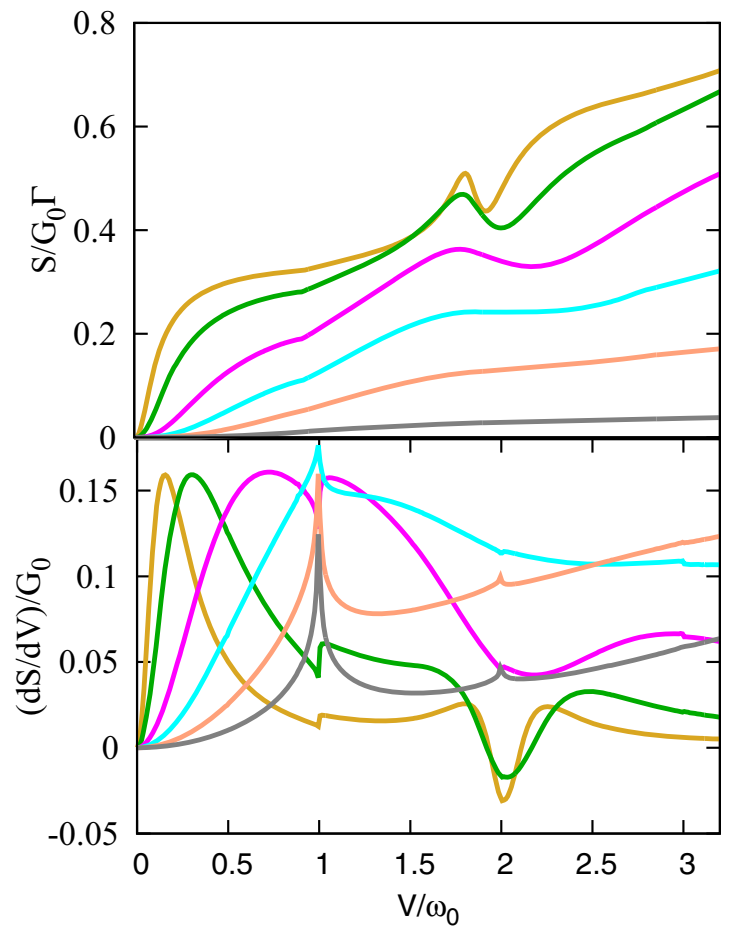

FIG. 10. (Color online) Zero-frequency noise within DTA for the same parameters as in Fig. 8. The lower panel corresponds to the differential noise $\partial S / \partial V$. From top to bottom in the upper panel $\Gamma=0.1,0.2,0.5,1.0,2.0$, and $4.0 \omega_{0}$.

observed in the first inset of Fig. 9, the initial step-up feature for the symmetric case evolves into a step down as $\tilde{\epsilon}$ approaches $\omega_{0} / 2$, where the elastic resonance condition $V / 2=\tilde{\epsilon}$ coincide with the inelastic threshold. In Fig. 9 we compare the DTA results with those of the EOM method obtained in Ref. [43]. As can be observed, there is a remarkable agreement between the two methods in this range of parameters. Additionally, the results exhibit a second inelastic threshold at $V \sim 2 \omega_{0}$, which can be more clearly appreciated when $\tilde{\epsilon}$ is shifted from zero energy (see the right inset of Fig. 9). This feature cannot be recovered by other methods like PTA, SPA, or ISA.

We next analyze the results for the current noise within DTA. Figure 10 shows the noise and the differential noise, $\partial S / \partial V$, as a function of voltage for increasing values of $\Gamma$ at fixed $g$ for the same parameter choice as in Fig. 8 for the differential conductance. There is an overall behavior of $\partial S / \partial V$ which is maintained for all values of $\Gamma$ : It starts from a zero value at $V=0$ as it corresponds to a perfect transmitting channel at zero temperature; there is then a maximum at around $V \sim 2 \Gamma$ followed by a decay as expected for a Lorentzian resonance. In addition the noise exhibits features at $V \sim$ $n \omega_{0}$ as the conductance. However, there appear interesting differences. For instance, the feature at the inelastic threshold at $V \sim \omega_{0}$ evolves as a function of $\Gamma$ from a step up at small values to a step down at intermediate ones and eventually again to a step up at large $\Gamma$. As in the case of the conductance the features at large $\Gamma$ are masked by the logarithmic singularities in the real part of the self-energies and can only be identified by analyzing the behavior of the noise on the neighborhood of the inelastic threshold. This double change of sign in the

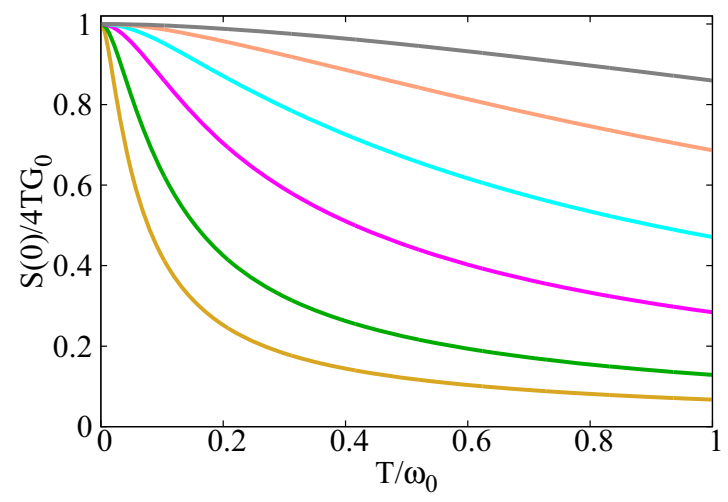

FIG. 11. (Color online) Thermal noise as a function of temperature within DTA for the same parameters as in Figs. 8 and 10. The noise is normalized as $S(0) / 4 T G_{0}$ in such a way as to illustrate the fulfillment of the FDT in the zero-temperature limit.

step is qualitatively in agreement with previous analysis of the noise in the perturbative regime [36-38] and which has been partially confirmed by experimental results for transport through small molecules [58].

As a final issue we discuss the zero-bias limit for the noise. In this limit the noise is purely due to thermal fluctuations and one should recover the fluctuation-dissipation theorem (FDT), stating that $S(0)=4 T G(0)$. This can be proved analytically within DTA (and also for the DSPA), as shown in Appendix A. Figure 11 illustrates the behavior of the thermal noise as a function of temperature for increasing values of $\Gamma$ at fixed $g$. As can be observed, all curves converge to the value $S(0) / 4 T G_{0}=$ 1 in the zero-temperature limit, thus indicating the fulfillment of FDT within this approach.

\section{CONCLUSION}

In this work we have presented and analyzed a theoretical approach for the nonequilibrium transport properties of nanoscale systems coupled to metallic electrodes with strong electron-phonon interactions. We have shown that this method, which we have called DTA, provides analytical expressions for the system GFs which are as simple as previous analytical approximations for the polaronic regime like SPA and PTA. We show, however, that the DTA eliminates the more remarkable pathologies of these two previous approximations in the low-energy (SPA) and high-energy (PTA) regimes. By comparison with other methods we have shown that DTA additionally reproduces the correct behavior in the crossover regime, $\lambda^{2} / \omega_{0} \lesssim \Gamma$. Only in the limit $\lambda^{2} / \omega_{0} \Gamma \ll 1$ does this approximation progressively deviate from the results provided by perturbation theory in the electron-phonon coupling. Some exact known limits of the model like the fully empty or fully occupied dot case are also recovered within DTA.

On the other hand, we have shown that DTA provides results for the current and the differential conductance in good agreement with results from other more elaborate methods, including numerically exact methods like diagrammatic Monte Carlo. In addition, DTA can be formulated in a way which allows us to extract the noise properties and, more generally, the FCS of the model. We have provided an analysis of the 
main features of the voltage-dependent shot noise and also of the thermal noise. For this last case we have shown analytically that DTA fulfills the fluctuation-dissipation theorem. We have also demonstrated that DTA satisfies current and noise conservation laws. This is a remarkable property in view of the simplicity of the approximation and its non-self-consistent character [59].

For future applications, the simplicity of the method could allow to address more complex situations like the nonstationary response of the model as studied in Refs. [42,60]. On the other hand, although the method has been derived for the more simple single model, the same ideas could be, in principle, extended to models including several dot levels coupled to multiple phonon modes like the one discussed in Ref. [61]. One can also envisage improving the present approximation by including nonequilibrium effects in the phonon distribution, as discussed, for instance, in Ref. [31].

\section{ACKNOWLEDGMENTS}

We thank A. Komnik, A. Zazunov, and R. Avriller for very useful discussions. We are in debt to K. F. Albrecht for sending us the quantum Monte Carlo data for the comparisons. We also thank Spanish MINECO for financial support under project FIS2011-26516.

\section{APPENDIX: EXPRESSIONS FOR THE NOISE AND THE CGF IN THE DIFFERENT APPROXIMATIONS}

Within PTA the CGF has the same expression as for a noninteracting system [41]

$$
\begin{aligned}
\ln \chi(v)= & \int \frac{d \omega}{2 \pi} \ln \left\{1+T(\omega)\left[\left(e^{i v}-1\right) f_{L}(\omega)\left(1-f_{R}(\omega)\right)\right.\right. \\
& \left.\left.+\left(e^{-i v}-1\right) f_{R}(\omega)\left(1-f_{L}(\omega)\right)\right]\right\}
\end{aligned}
$$

with a renormalized transmission $T(\omega)$ given by $T(\omega)=4 \Gamma_{L} \Gamma_{R} /\left(f^{-2}(\omega)+\Gamma^{2}\right)$, where

$$
f(\omega) \equiv \sum_{k=-\infty}^{\infty} \alpha_{k}\left(\frac{n_{0}}{\omega-\tilde{\epsilon}+k \omega_{0}}+\frac{1-n_{0}}{\omega-\tilde{\epsilon}-k \omega_{0}}\right)
$$

For the other approximations (DTA and DSPA), the expressions are slightly more involved. We will first analyze the DTA case. The Keldysh GFs within this approximation, including the counting field, can be written as

$$
\begin{aligned}
& G_{\mathrm{DTA}}^{+-(v)}(\omega)=-\sum_{k=-\infty}^{\infty} \alpha_{k} \frac{\tilde{\Sigma}_{L}^{+-}\left(\omega^{\prime}\right) e^{i v}+\tilde{\Sigma}_{R}^{+-}\left(\omega^{\prime}\right)}{\tilde{\mathfrak{D}}^{(v)}\left(\omega^{\prime}\right)} \\
& G_{\mathrm{DTA}}^{-+(v)}(\omega)=-\sum_{k=-\infty}^{\infty} \alpha_{-k} \frac{\tilde{\Sigma}_{L}^{-+}\left(\omega^{\prime}\right) e^{-i v}+\tilde{\Sigma}_{R}^{-+}\left(\omega^{\prime}\right)}{\tilde{\mathfrak{D}}^{(\nu)}\left(\omega^{\prime}\right)},
\end{aligned}
$$

where $\omega^{\prime}=\omega+k \omega_{0}$ and

$$
\begin{aligned}
\mathfrak{D}^{(\nu)}(\omega)= & \tilde{\mathfrak{D}}(\omega)+\tilde{\Sigma}_{0 L}^{-+}(\omega) \tilde{\Sigma}_{0 R}^{+-}(\omega)\left(e^{-i \nu}-1\right) \\
& +\tilde{\Sigma}_{0 L}^{+-}(\omega) \tilde{\Sigma}_{0 R}^{-+}(\omega)\left(e^{i v}-1\right) .
\end{aligned}
$$

By using Eq. (11) for the current between the dot and the right electrode one obtains

$$
I_{\mathrm{DTA}}=\int \frac{d \omega}{2 \pi} \frac{\tilde{\Sigma}_{0 R}^{+-}(\omega) \tilde{\Sigma}_{0 L}^{-+}(\omega)-\tilde{\Sigma}_{0 R}^{-+}(\omega) \tilde{\Sigma}_{0 L}^{+-}(\omega)}{\tilde{\mathfrak{D}}(\omega)},
$$

which is clearly antisymmetric with respect to the interchange of $L$ and $R$, thus indicating current conservation within DTA.

In an analogous way, the voltage-dependent noise can be straightforwardly computed from Eq. (13),

$$
\begin{aligned}
S_{\mathrm{DTA}}= & \int \frac{d \omega}{\pi} \frac{\tilde{\Sigma}_{0 R}^{+-}(\omega) \tilde{\Sigma}_{0 L}^{-+}(\omega)+\tilde{\Sigma}_{0 R}^{-+}(\omega) \tilde{\Sigma}_{0 L}^{+-}(\omega)}{\tilde{\mathfrak{D}}(\omega)} \\
& -\left[\frac{\tilde{\Sigma}_{0 R}^{+-}(\omega) \tilde{\Sigma}_{0 L}^{-+}(\omega)-\tilde{\Sigma}_{0 R}^{-+}(\omega) \tilde{\Sigma}_{0 L}^{+-}(\omega)}{\tilde{\mathfrak{D}}(\omega)}\right]^{2}
\end{aligned}
$$

In addition to current conservation it is interesting to check whether this approximation fulfills the fluctuationdissipation theorem, which relates the thermal noise at zero bias with the linear conductance by $S(0)=4 T G(0)$. At zero voltage $\Sigma_{0 L}^{\alpha \beta}(\omega) / \Gamma_{L}=\Sigma_{0 R}^{\alpha \beta}(\omega) / \Gamma_{R}(\alpha, \beta=+-)$. Then the zero voltage noise can be written as

$$
S_{\mathrm{DTA}}(0)=\int \frac{d \omega}{\pi} \frac{\tilde{\Sigma}_{0 R}^{+-}(\omega) \tilde{\Sigma}_{0 L}^{-+}(\omega)+\tilde{\Sigma}_{0 R}^{-+}(\omega) \tilde{\Sigma}_{0 L}^{+-}(\omega)}{\tilde{\mathfrak{D}}(\omega)} .
$$

On the other hand, the zero bias conductance can be computed from Eq. (A5), yielding

$$
G_{\mathrm{DTA}}(0)=\frac{1}{4 T} \int \frac{d \omega}{\pi} \frac{\tilde{\Sigma}_{0 R}^{+-}(\omega) \tilde{\Sigma}_{0 L}^{-+}(\omega)+\tilde{\Sigma}_{0 R}^{-+}(\omega) \tilde{\Sigma}_{L}^{+-}(\omega)}{\tilde{\mathfrak{D}}(\omega)} .
$$

Comparison with Eq. (A7) clearly shows the fulfillment of the fluctuation-dissipation theorem within DTA.

Similar expressions can be derived for the DSPA. First, the current flowing through the right electrode can be written as

$$
I_{R, \mathrm{DSPA}}=\int \frac{d \omega}{2 \pi} \frac{\Sigma_{0 L}^{+-}(\omega) \tilde{\Sigma}_{0 R}^{-+}(\omega)-\Sigma_{0 L}^{-+}(\omega) \tilde{\Sigma}_{0 R}^{+-}(\omega)}{(\omega-\tilde{\epsilon})^{2}+\Gamma^{2}} .
$$

It is important to notice that this expression is not symmetric with respect to $L$ and $R$ exchange when the level $\tilde{\epsilon}$ is displaced from zero. This absence of symmetry breaks the current conservation for bias voltages bigger than $\omega_{0}$.

In the same way the noise can be computed analytically within DSPA,

$$
\begin{aligned}
S_{R, \mathrm{DSPA}}= & \int \frac{d \omega}{\pi} \frac{\tilde{\Sigma}_{0 R}^{+-}(\omega) \Sigma_{0 L}^{-+}(\omega)+\tilde{\Sigma}_{0 R}^{-+}(\omega) \Sigma_{0 L}^{+-}(\omega)}{\Gamma^{2}+(w-\tilde{\epsilon})^{2}} \\
& +4 \Gamma_{L} \Gamma_{R}\left(f_{L}(\omega)-f_{R}(\omega)\right) \\
& \times \frac{\tilde{\Sigma}_{0 R}^{+-}(\omega) \Sigma_{0 L}^{-+}(\omega)-\tilde{\Sigma}_{0 R}^{-+}(\omega) \Sigma_{0 L}^{+-}(\omega)}{\left[\Gamma^{2}+(w-\tilde{\epsilon})^{2}\right]^{2}},
\end{aligned}
$$

which again exhibits explicitly the breaking of left-right symmetry, i.e., noise is not conserved in general within DSPA. It can be shown, however, that within this approximation the fluctuation-dissipation theorem is fulfilled. 
[1] M. Galperin, M. A. Ratner, and A. Nitzan, J. Phys.: Condens. Matter 19, 103201 (2007).

[2] N. Agraï, C. Untiedt, G. Rubio-Bollinger, and S. Vieira, Phys. Rev. Lett. 88, 216803 (2002).

[3] E. M. Weig, R. H. Blick, T. Brandes, J. Kirschbaum, W. Wegscheider, M. Bichler, and J. P. Kotthaus, Phys. Rev. Lett. 92, 046804 (2004).

[4] R. Leturcq et al., Nat. Phys. 5, 327 (2009).

[5] H. Park et al., Nature 407, 57 (2000).

[6] R. H. M. Smit et al., Nature 419, 906 (2002).

[7] N. B. Zhitenev, H. Meng, and Z. Bao, Phys. Rev. Lett. 88, 226801 (2002).

[8] F. Ortmann, F. Bechstedt, and K. Hannewald, Phys. Rev. B 79, 235206 (2009).

[9] F. Ortmann and S. Roche, Phys. Rev. B 84, 180302 (2011).

[10] T. Holstein, Ann. Phys. (N. Y.) 8, 343 (1959).

[11] D. C. Langreth, Phys. Rev. B 1, 471 (1970).

[12] L. Glazman and R. Shekhter, Zh. Eksp. Teor. Fiz. 94, 292 (1988) [Sov. Phys. JETP 67, 163 (1988)].

[13] N. S. Wingreen, K. W. Jacobsen, and J. W. Wilkins, Phys. Rev. B 40, 11834 (1989).

[14] K. Flensberg, Phys. Rev. B 68, 205323 (2003).

[15] A. Mitra, I. Aleiner, and A. J. Millis, Phys. Rev. B 69, 245302 (2004).

[16] A. C. Hewson and D. Meyer, J. Phys.: Condens. Matter 14, 427 (2002).

[17] G. S. Jeon, T.-H. Park, and H.-Y. Choi, Phys. Rev. B 68, 045106 (2003).

[18] P. S. Cornaglia, H. Ness, and D. R. Grempel, Phys. Rev. Lett. 93, 147201 (2004).

[19] L. Arrachea and M. J. Rozenberg, Phys. Rev. B 72, 041301(R) (2005).

[20] L. Mühlbacher and E. Rabani, Phys. Rev. Lett. 100, 176403 (2008).

[21] K. F. Albrecht, H. Wang, L. Mühlbacher, M. Thoss, and A. Komnik, Phys. Rev. B 86, 081412 (2012).

[22] A. Jovchev and F. B. Anders, Phys. Rev. B 87, 195112 (2013).

[23] R. Hützen, S. Weiss, M. Thorwart, and R. Egger, Phys. Rev. B 85, 121408 (2012).

[24] M. A. Laakso, D. M. Kennes, S. G. Jakobs, and V. Meden, New J. Phys. 16, 023007 (2014).

[25] J. K. Viljas, J. C. Cuevas, F. Pauly, and M. Hafner, Phys. Rev. B 72, 245415 (2005)

[26] R. Egger and A. O. Gogolin, Phys. Rev. B 77, 113405 (2008)

[27] O. Entin-Wohlman, Y. Imry, and A. Aharony, Phys. Rev. B 80, 035417 (2009).

[28] T. Frederiksen, M. Brandbyge, N. Lorente, and A. P. Jauho, Phys. Rev. Lett. 93, 256601 (2004).

[29] L. de la Vega, A. Martín-Rodero, N. Agraït and A. Levy Yeyati, Phys. Rev. B 73, 075428 (2006).

[30] L. K. Dash, H. Ness, and R. W. Godby, Phys. Rev. B 84, 085433 (2011).

[31] M. Galperin, A. Nitzan, and M. A. Ratner, Phys. Rev. B 73, 045314 (2006).

[32] S. Braig and K. Flensberg, Phys. Rev. B 68, 205324 (2003).
[33] J. Koch and F. von Oppen, Phys. Rev. Lett. 94, 206804 (2005).

[34] A. Martín-Rodero, A. Levy Yeyati, F. Flores, and R. C. Monreal, Phys. Rev. B 78, 235112 (2008).

[35] B. Dong, G. H. Ding, and X. L. Lei, Phys. Rev. B 88, 075414 (2013).

[36] R. Avriller and A. Levy Yeyati, Phys. Rev. B 80, 041309(R) (2009).

[37] T. L. Schmidt and A. Komnik, Phys. Rev. B 80, 041307(R) (2009).

[38] F. Haupt, T. Novotny, and W. Belzig, Phys. Rev. Lett. 103, 136601 (2009).

[39] D. F. Urban, R. Avriller, and A. Levy Yeyati, Phys. Rev. B 82, 121414 (2010).

[40] M. Galperin, A. Nitzan, and M. A. Ratner, Phys. Rev. B 74, 075326 (2006).

[41] S. Maier, T. L. Schmidt, and A. Komnik, Phys. Rev. B 83, 085401 (2011).

[42] K. F. Albrecht, A. Martín-Rodero, R. C. Monreal, L. Muhlbacher and A. Levy Yeyati, Phys. Rev. B 87, 085127 (2013).

[43] R. C. Monreal and A. Martín-Rodero, Phys. Rev. B 79, 115140 (2009).

[44] R. C. Monreal, F. Flores, and A. Martín-Rodero, Phys. Rev. B 82, 235412 (2010).

[45] A. Zazunov and T. Martin, Phys. Rev. B 76, 033417 (2007).

[46] J. König, J. Schmid, H. Schoeller, and G. Schon, Phys. Rev. B 54, 16820 (1996).

[47] P. Roura-Bas, L. Tosi, and A. A. Aligia, Phys. Rev. B 87, 195136 (2013).

[48] R. Seoane, M.S. thesis, Universidad Autónoma de Madrid, 2013.

[49] I. G. Lang and Y. A. Firsov, JETP 16, 1301 (1963).

[50] G. Mahan, Many Particle Physics (Plenum Press, New York, 1981).

[51] L. V. Keldysh, Zh. Eksp. Teor. Fiz. 47, 1515 (1964) [Sov. Phys. JETP 20, 1018 (1965)].

[52] Y. V. Nazarov, ed. Quantum Noise in Mesoscopic Physics (Springer, Berlin 2003).

[53] A. O. Gogolin and A. Komnik, Phys. Rev. B 73, 195301 (2006).

[54] Y. Meir and N. S. Wingreen, Phys. Rev. Lett. 68, 2512 (1992).

[55] A similar philosophy is the starting point for more general models like those of the following: J. Bonca and S. A. Trugman, Phys. Rev. Lett. 75, 2566 (1995); M. Galperin, A. Nitzan, and M. A. Ratner, Phys. Rev. B 78, 125320 (2008); A. J. White and M. Galperin, Phys. Chem. Chem. Phys 14, 13809 (2012).

[56] A. C. Hewson and D. Newns, J. Phys. C 12, 1665 (1979).

[57] A. Levy Yeyati, A. Martín-Rodero and F. Flores, Phys. Rev. Lett. 71, 2991 (1993).

[58] M. Kumar, R. Avriller, A. L. Yeyati and J. M. van Ruitenbeek, Phys. Rev. Lett. 108, 146602 (2012).

[59] For a detailed analysis of the interplay between self-consistency and conservation laws in the perturbative regime see K. S. Thygesen and A. Rubio, Phys. Rev. B 77, 115333 (2008); H. Mera, M. Lannoo, N. Cavassilas, and M. Bescond, ibid. 88 , 075147 (2013).

[60] E. Perfetto and G. Stefanucci, Phys. Rev. B 88, 245437 (2013).

[61] T. Brandes and B. Kramer, Phys. Rev. Lett. 83, 3021 (1999). 